\title{
TRACE CONTAMINATION MEASUREMENTS USING HEAVY ION \\ BACKSCATTERING SPECTROMETRY
}

\author{
J. A. KNAPP, J. C. BANKS AND D. K. BRICE \\ Sandia National Laboratories, Albuquerque, NM 87185
}

\section{ABSTRACT}

Heavy Ion Backscattering Spectrometry (HIBS) is a tool for measuring surface contamination at levels much lower than previously achievable with ion beam analyses. Many areas of materials research and development require very low levels of impurities, with advanced microelectronics as the best example. HIBS uses low-energy, heavy ions for analysis, taking advantage of the increased backscattering yield which can be obtained from high $\mathrm{Z}$ ion beams at low energy. Background due to increased yield from the substrate is eliminated by a thin carbon foil as part of the detector, with a thickness chosen to range out ions scattered from the substrate and allow only ions scattered from impurities heavier than the substrate to reach the detector. Our research system, using 150-200 $\mathrm{keV} \mathrm{N}{ }^{+}$beams and a single time-of-flight detector optimized for large solid angle, has achieved a sensitivity ranging from $\sim 5 \times 10^{10}$ atoms $/ \mathrm{cm}^{2}$ for $\mathrm{Fe}$ to $\sim 1 \times 10^{9}$ atoms $/ \mathrm{cm}^{2}$ for $\mathrm{Au}$ on $\mathrm{Si}$, without preconcentration. A stand-alone HIBS prototype which we have developed for SEMATECH is expected to reach detection limits of $\sim 5 \times 10^{9}$ atoms $/ \mathrm{cm}^{2}$ for $\mathrm{Fe}$ and $\sim 1 \times 10^{8}$ atoms $/ \mathrm{cm}^{2}$ for $\mathrm{Au}$ on Si. In this paper we discuss in detail the factors which affect sensitivity for HIBS.

\section{INTRODUCTION}

The continuing increase in cleanliness requirements for the microelectronics industry is well known. By the turn of the century, very large scale integrated circuit processing is expected to require contamination levels well below $1 \times 10^{9}$ atoms $/ \mathrm{cm}^{2}$ in both starting materials and introduced by processing. ${ }^{1}$ There are several approaches being explored for measuring these extremely low levels of contaminants; a good example is Total reflection X-Ray Fluorescence (TXRF), already available as a commercial tool and widely used in the industry. TXRF is reaching its limits at around $1 \times 10^{10}$ atoms $/ \mathrm{cm}^{2}$ levels for many elements such as $\mathrm{Fe}$ and $\mathrm{Cu}$, and for other elements may be limited to $1 \times 10^{12}$ atoms $/ \mathrm{cm}^{2}$ or worse. The sensitivity of stand-alone TXRF instruments may be extended through the use of pre-concentration, but this introduces considerable uncertainty. Another disadvantage of TXRF is that only a subset of the periodic chart is examined with a single setup; it is necessary to change X-ray sources to measure both $\mathrm{Fe}$ and $\mathrm{Br}$, for example. However, when combined with a synchrotron storage ring as the X-ray source, TXRF may reach sensitivities of $1 \times 10^{8}$ atoms $/ \mathrm{cm}^{2}$ or better.

Heavy Ion Backscattering Spectrometry (HIBS) is an ion beam analysis technique using heavy, low energy ions to detect very low levels of surface contamination. ${ }^{2-7}$ By taking advantage of the greatly increased scattering cross-section for such ion beams and eliminating unwanted substrate scattering with a thin screening foil as part of the detector, we have achieved sensitivities to impurities on $\mathrm{Si}$ ranging from $\sim 5 \times 10^{10}$ atoms $/ \mathrm{cm}^{2}$ for $\mathrm{Fe}$ to $\sim 1 \times 10^{9}$ atoms $/ \mathrm{cm}^{2}$ for $\mathrm{Au}$, without preconcentration. A new system, under construction in a collaboration with 


\section{DISCLAIMER}

This report was prepared as an account of work sponsored by an agency of the United States Government. Neither the United States Government nor any agency thereof, nor any of their employees, makes any warranty, express or implied, or assumes any legal liability or responsibility for the accuracy, completeness, or usefulness of any information, apparatus, product, or process disclosed, or represents that its use would not infringe privately owned rights. Reference herein to any specific commercial product, process, or service by trade name, trademark, manufacturer, or otherwise does not necessarily constitute or imply its endorsement, recommendation, or favoring by the United States Government or any agency thereof. The views and opinions of authors expressed herein do not necessarily state or reflect those of the United States Government or any agency thereof. 


\section{DISCLAIMER}

Portions of this document may be illegible in electronic image products. Images are produced from the best available original document. 
SEMATECH, is expected to achieve detection limits on $\mathrm{Si}$ of $\sim 5 \times 10^{9}$ atoms $/ \mathrm{cm}^{2}$ for $\mathrm{Fe}$ and $\sim 1 \times 10^{8}$ atoms $/ \mathrm{cm}^{2}$ for Au. Since HIBS as an ion backscattering technique is standardless and has no matrix effects, it will be useful not only as a standalone tool for contamination surveys and quantification, but also for benchmarking standard samples for other tools. HIBS also has the advantages of measuring all elements above $\mathrm{Ar}$ in a single spectrum and the capability of measuring patterned or rough surfaces.

\section{EXPERIMENTAL}

HIBS, which is most useful for trace analysis of heavy impurities on the surface of a light substrate, is a modification of Rutherford backscattering spectrometry (RBS). RBS is typically performed using a 1-2 $\mathrm{MeV} \mathrm{He}^{+}$ion beam for the analysis, resulting in a sensitivity of $\sim 10^{13}$ atoms $/ \mathrm{cm}^{2}$ to impurities at or near the surface, limited by system noise and pulse pileup. In an ion backscattering experiment the yield of counts from a particular target for a given incident beam energy is given by Rutherford's formula ${ }^{8}$ for the differential scattering cross-section, as transformed from center-of-mass to a laboratory frame of reference by Darwin ${ }^{9}$ :

$$
\frac{d \sigma}{d \Omega}=\left(\frac{Z_{1} Z_{2} e^{2}}{2 E \sin ^{2} \theta}\right)^{2} \frac{\left\{\cos \theta+\left[1-\left(\frac{M_{1}}{M_{2}} \sin \theta\right)^{2}\right]^{\frac{1}{2}}\right\}^{2}}{\left[1-\left(\frac{M_{1}}{M_{2}} \sin \theta\right)^{2}\right]^{\frac{1}{2}}}
$$

where $Z_{1}$ and $Z_{2}$ are the atomic numbers of the projectile and target atoms, e is electron charge, and $E$ is the energy of the projectile immediately before scattering. The average scattering cross section, $\sigma$, is defined as

$$
\sigma=\frac{1}{\Omega} \int \frac{d \sigma}{d \Omega} d \Omega
$$

where the integration is over $\Omega$, the finite solid angle spanned by the detector. In the energy range of a few hundred $\mathrm{keV}$, as used in these experiments, the cross-sections are significantly modified by electronic screening effects ${ }^{10}$ and it is important to include an appropriate correction factor for quantitative results. We use the Andersen formulation ${ }^{11}$, which leads to a screened cross section given by:

$$
\frac{d \sigma / d \Omega}{(d \sigma / d \Omega)_{R}}=\frac{1}{1+V_{1}^{L J} / E_{c m}}
$$

where

$$
V_{1}^{L I}=48.73 Z_{1} Z_{2}\left(Z_{1}^{2 / 3}+Z_{2}^{2 / 3}\right)^{1 / 2} \mathrm{eV}
$$


These equations show that the backscattering yield is proportional to the square of the atomic number of the analyzing beam and inversely proportional to the square of its energy, so a greatly enhanced yield can be obtained by using a higher $Z$ ion beam at lower energy. Although this yield enhancement is well known, it is not widely exploited because pileup due to yield from the lower mass substrate overwhelms the signal from ions scattered by heavy trace surface impurities. (Pileup occurs in an energy detector when multiple low-energy signals arrive simultaneously at a detector, such that they are indistinguishable from a single, higher-energy event). The key for HIBS is to essentially eliminate the pileup background by using a ranging foil as part of the detector which allows only particles backscattered from species heavier than the substrate to reach the detector.

We developed a time-of-flight ion detector for use with HIBS, optimizing the design to achieve as high a sensitivity as possible. ${ }^{5,6}$ The design allows up to three detectors to be arrayed around the analysis beam to give correspondingly higher sensitivity. A second detector will be added to the research system shortly, while the system being built for SEMATECH will have an arrangement of three parallel detectors, along with a larger beam spot, which also improves the sensitivity.

Figure 1 shows a diagram of the time-of-flight detector, which uses a large area stop detector and a relatively short $12 \mathrm{~cm}$. flight path to maximize solid angle, while keeping energy resolution within acceptable bounds. The $25 \mathrm{~nm} \mathrm{C}$ foil serves two purposes: first, it screens out the particles scattered from the light substrate, as discussed above, and second, it provides a burst of electrons from those particles which are energetic enough to pass through. These electrons are accelerated and detected by a multi-channelplate (MCP) detector to provide a timing start pulse. The particles proceed along a $12 \mathrm{~cm}$ flight path to a second $\mathrm{MCP}$, where they are also detected, providing the timing stop pulse. Since it was desirable to maximize the solid angle for improving sensitivity, the angle subtended by the ion MCP is relatively large, which leads to kinematic broadening. That is, the kinematic factor (energy) for backscattering from each mass changes somewhat from one side of the plate to the other. The angles of both the foil and the ion MCP were chosen by computer simulation during the design phase to minimize this broadening. More

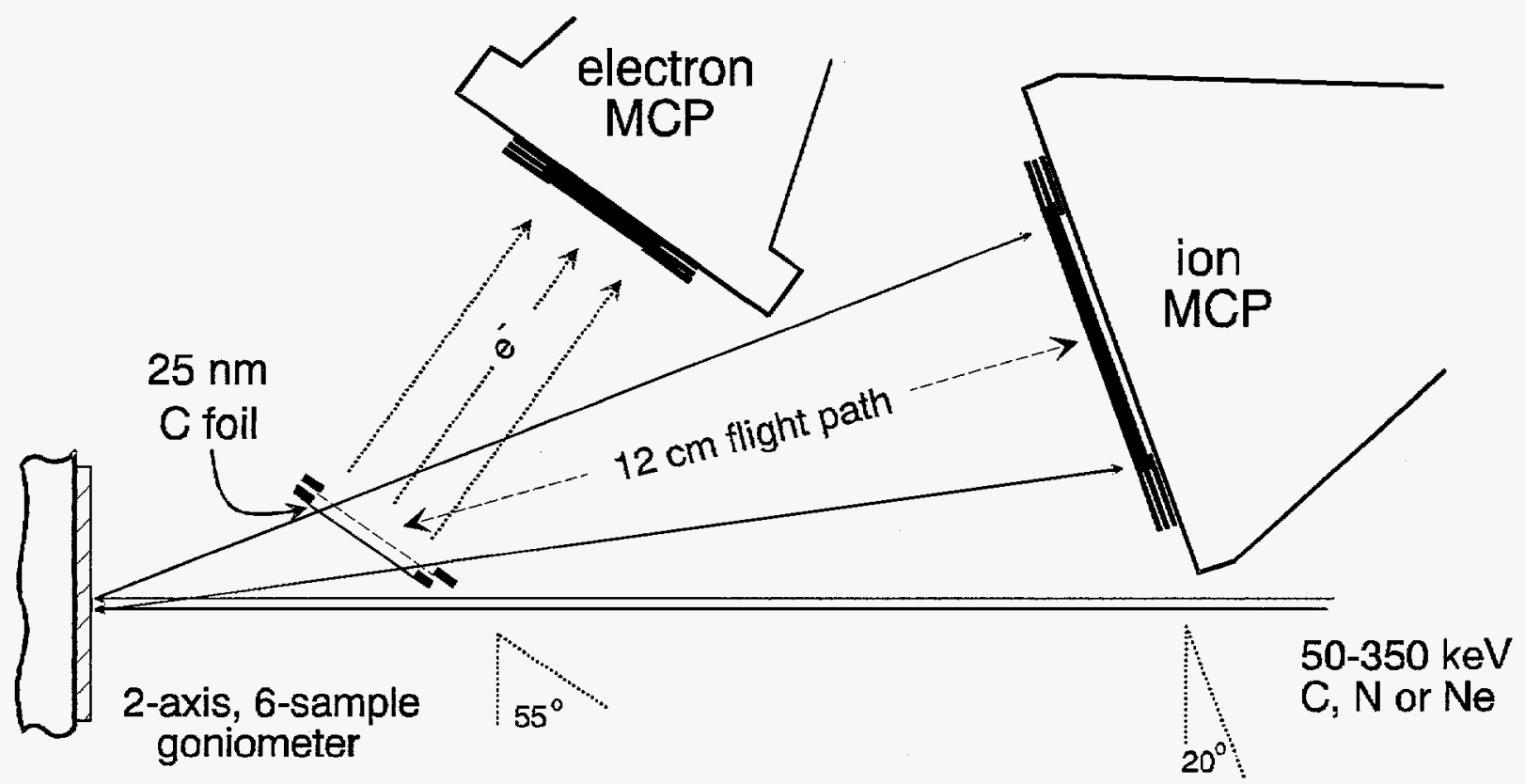

Figure 1. Schematic of the Time-of-Flight HIBS detector. 


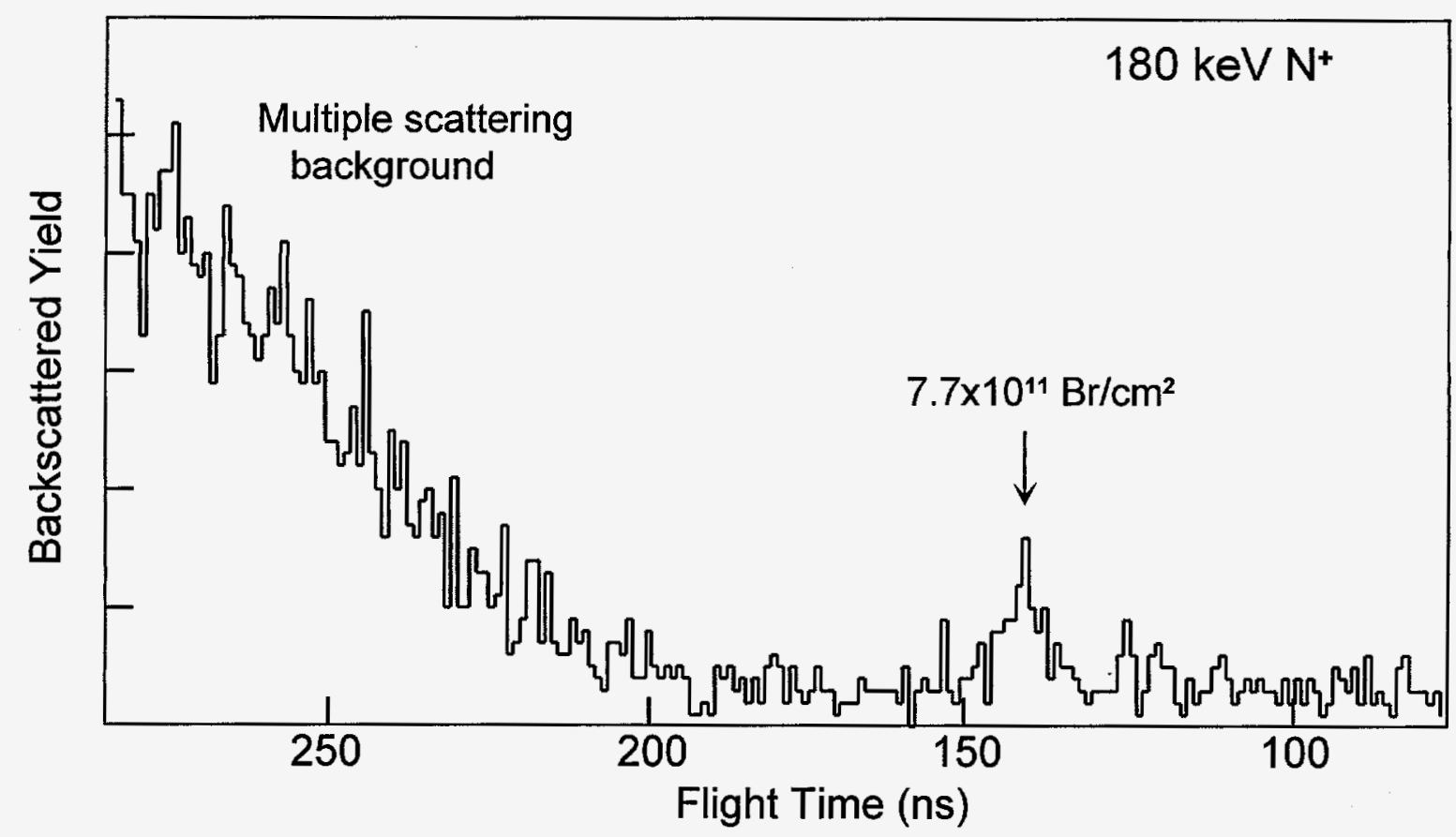

Figure 2. Time-of-Flight HIBS spectrum obtained from a Br-contaminated Si wafer. A $180 \mathrm{keV}$ $\mathrm{N}^{+}$beam was used with a total exposure of $60 \mu \mathrm{C}$, in a $2 \mathrm{~mm}$ spot.

details of the design and other parts of the system are presented elsewhere. ${ }^{5,6}$

Figure 2 shows a typical HIBS spectrum obtained with the research system. The sample was a clean $\mathrm{Si}$ wafer which was exposed to a plasma during an equipment test. The $\mathrm{Br}$ contamination which is detected is due to the past history of the chamber, which had been used with a Brcontaining plasma. The spectrum illustrates the low levels of contaminants which can be readily seen, as well as some of the features which limit the sensitivity of the technique. In particular, the background at larger flight times (low energy) is a fundamental limit, due to multiple scattering in the $\mathrm{Si}$ substrate.

\section{SENSITIVITY}

We now discuss the factors which affect the ultimate sensitivity which can be achieved with HIBS. The most obvious factor has already been mentioned in the discussion of Eqs. 1-4: the yield, and hence the potential sensitivity, increases with decreasing beam energy and increasing beam $Z$. Even though electron screening reduces this benefit at the low end, the cross-section still increases monotonically with lower energy. On the other hand, there are a number of competing factors which become worse at lower energy and thus offset the gains in cross section. The choices of foil thickness, beam species and beam energy to achieve the best possible sensitivity for a particular problem depends on the combination of all factors.

\section{Sputtering by the analysis beam}

Sputtering of the sample surface by the analysis beam gives the ultimate limit to the statistics which can be obtained, and hence the sensitivity. An impurity cannot be measured if the act of measurement removes it before the spectrum is complete, although a lower level can be 
measured if a larger beam spot is used. The rate of sputtering will depend on the state of the impurity: particles will sputter differently than widely dispersed, low concentration layers, so it is difficult to predict the limit in advance. Simple experiments, wherein a sample was repeatedly analyzed on the same spot, demonstrated sputtering for our system and suggested that the rate for a sub-monolayer impurity was lower than predicted by a simple sputtering model using bulk sputtering rates. A more recent and comprehensive series of measurements with collaborators at Vanderbilt University have confirmed this: for the materials tested, sub-monolayer impurities are sputtered by the beam much more slowly than the underlying Si substrate. A detailed report on these results is in preparation. ${ }^{12}$ The conclusion is that sputtering by the analysis beam, although still the ultimate limit, will allow measurements down to much lower levels than would be possible if bulk sputtering rates applied.

\section{Detector efficiency}

The efficiency of the channelplates for detecting electrons; ions and neutrals in these energy ranges is not well known, nor is the electron-producing efficiency of the ranging foil. The channelplates also have a count rate limitation which may in turn limit the amount of beam current which can be used. Straggling in the foil is also an important effect, dominating the observed time resolution and limiting the mass resolution that can be achieved. The mass resolution of the research system, with its present $25 \mathrm{~nm}$ carbon foil, is about $1 \mathrm{amu}$ for Ti-V, 3 amu for $\mathrm{Cu}-\mathrm{Zn}$, and 18-10 amu for the heaviest elements. This peak broadening also affects sensitivity, since at lower detected energy the peak from a particular mass is spread over a broader range of background.

A more serious problem for sensitivity is scattering in the foil, which results in some ions exiting at angles which don't intercept the ion channelplate, reducing the effective solid angle of the detector. This effect is stronger at lower energy, offsetting the gains that can be obtained by going to lower beam energy. The problem is illustrated by TRIM $^{13}$ calculations of the distribution of trajectories of $\mathrm{N}$ particles passing through the foil at two different energies, shown in Fig. 3. The percentage of particles which remain in the acceptance angle of the ion MCP is much lower at low energy: the simulation shows $85 \%$ of the $160 \mathrm{kev}$ particles reach the MCP, but only $23 \%$ at $40 \mathrm{keV}$.

The effect of foil scattering will vary with beam species and energy, and of course will be different for different foil thicknesses. For a given foil, measurements of the detector efficiency

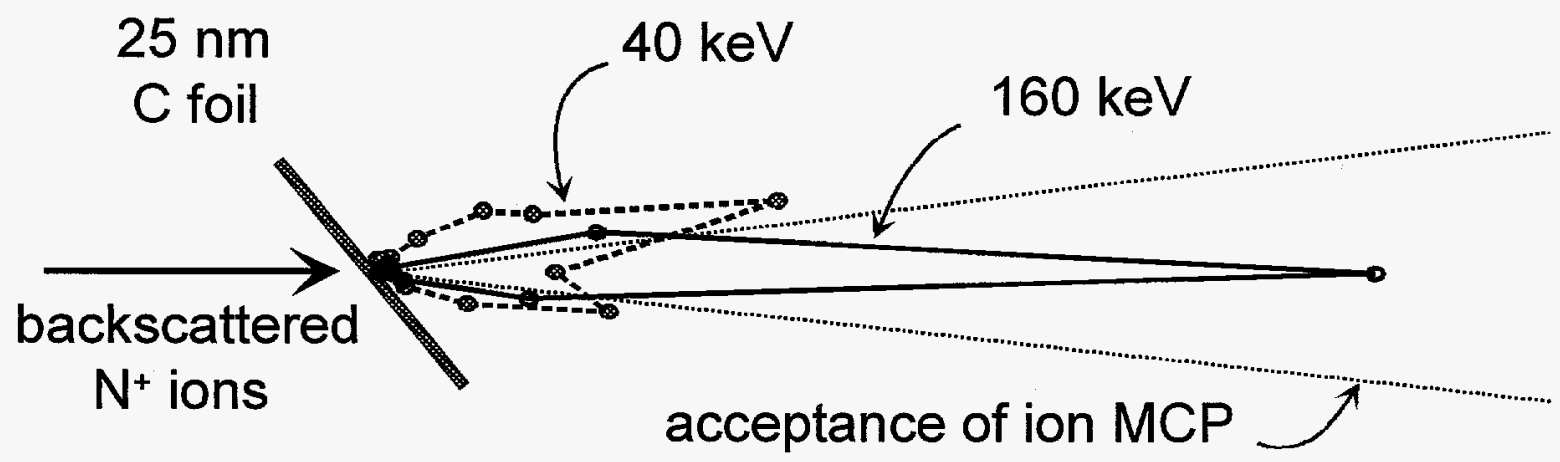

Figure 3. TRIM calculations of the angular distribution of $\mathrm{N}$ after passing through the screening foil. Distributions for two energies are shown: 40 and $160 \mathrm{keV}$. The counts plotted are those which are scattered within $\pm 15^{\circ}$ of the plane of the diagram. The dashed line shows the acceptance angle of the ion MCP. 


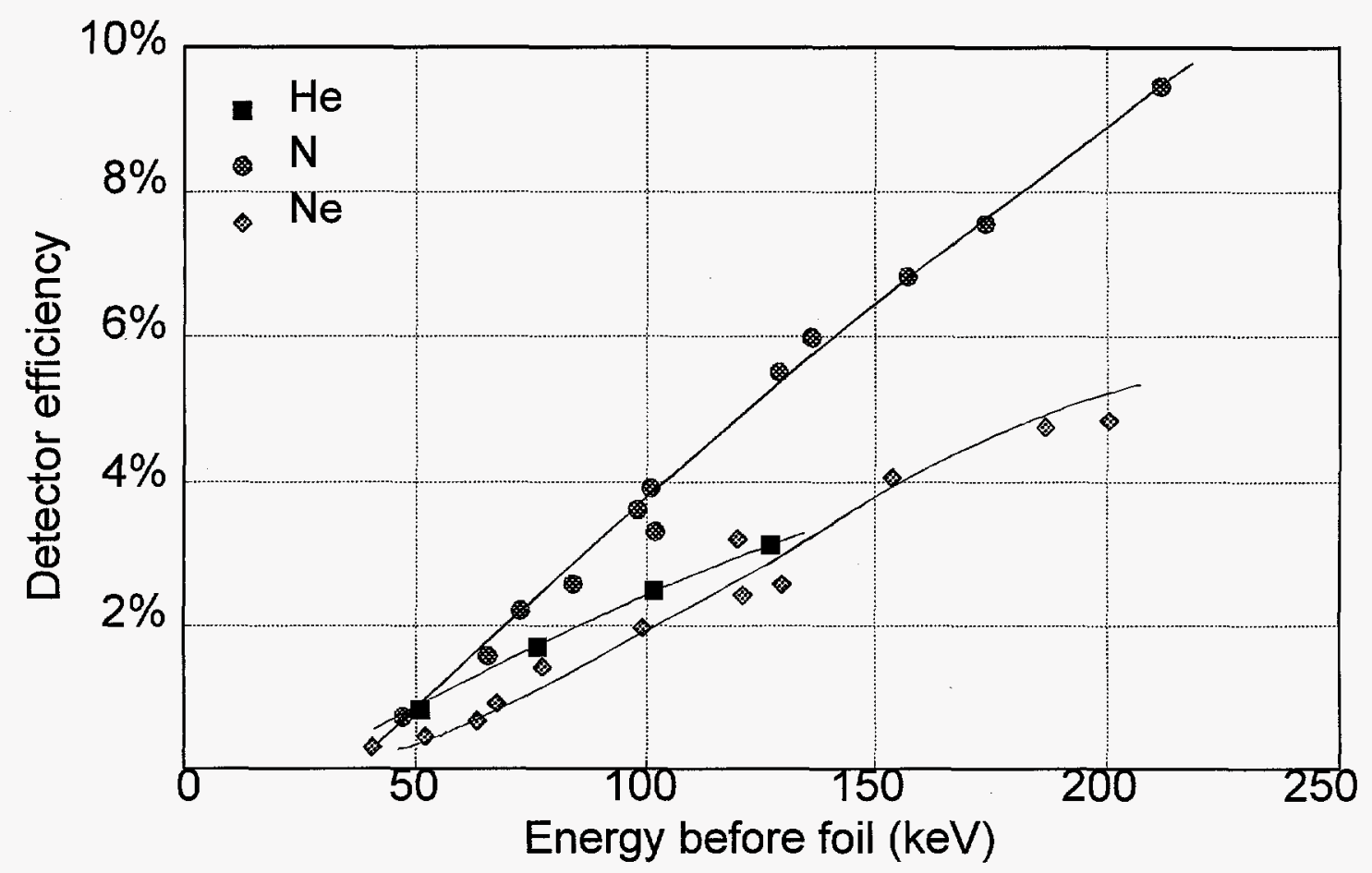

Figure 4. Detector efficiency for beams of $\mathrm{He}, \mathrm{N}$, and $\mathrm{Ne}$, using a $25 \mathrm{~nm} \mathrm{C}$ foil.

will show that some ions are detected more efficiently than others, primarily because of the relative amounts of foil scattering, but also due to details of electron production efficiency and channelplate detection efficiency. Such measurements for the research HIBS detector are shown in Fig. 4, where the overall efficiency of the detector for three beam species, $\mathrm{He}, \mathrm{N}$, and $\mathrm{Ne}$, are plotted versus the energy of the backscattered particles (i.e. energy before the foil). As can be seen, this configuration of the detector (with a $25 \mathrm{~nm}$ foil) is most efficient for $\mathrm{N}$ beams. Note that for He beams, this foil thickness is insufficient to screen out the scattering from the $\mathrm{Si}$ substrate, so the measured efficiency for He included the effects of a much higher overall count rate.

If the measured efficiency is then combined with the screened cross section from Eqs. 1-4, an effective cross section can be determined for each beam and target combination. Effective cross sections for $\mathrm{Fe}$ and $\mathrm{Au}$ in our detector using beams of $\mathrm{He}, \mathrm{N}$, and $\mathrm{Ne}$ are plotted in Fig. 5. This illustrates that the highest yield for most elements can be expected using a $\mathrm{N}$ beam with energies of $150-200 \mathrm{keV}$. The sensitivity which can be achieved, however, depends not only on the yield, but also the background, as will be discussed next.

An analytical model of the efficiency of time-of-flight ion detectors of this general configuration has been developed elsewhere ${ }^{14,15}$, and will be shortly applied to this detector design and the similar ones we are using in the SEMATECH system. The model will allow the prediction of optimum beam and energy conditions for a particular problem without having to physically measure the efficiency for all possibilities.

\section{Background}

The optimum condition for high sensitivity maximizes the ratio of peak yield to the square root of the background under the peak of interest. Achieving this may require operating with the thresholds for the MCP electronics at higher values to discriminate against system noise or low 


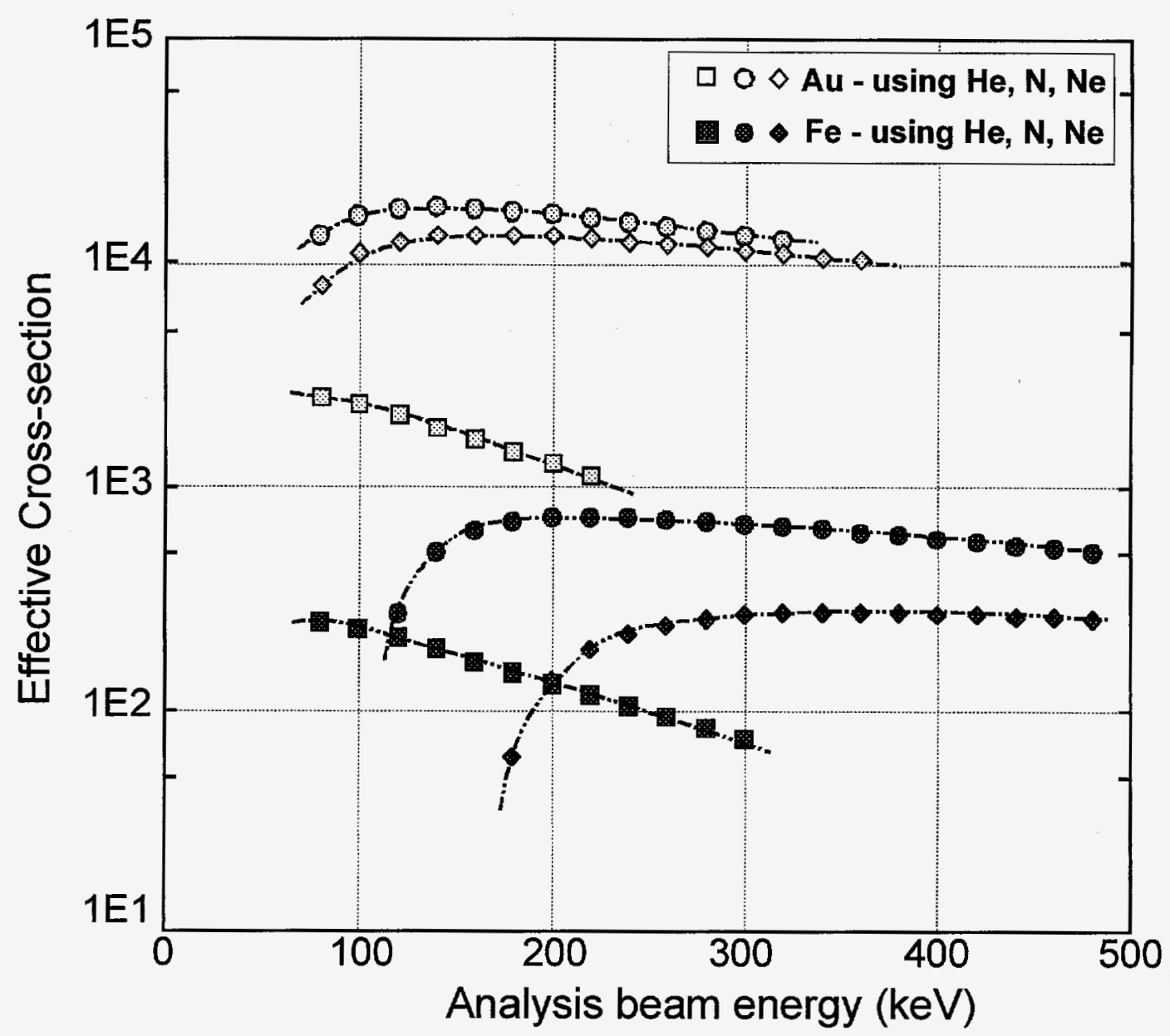

Figure 5. Cross sections for $\mathrm{Fe}$ and $\mathrm{Au}$, for beams of $\mathrm{He}, \mathrm{N}$, and $\mathrm{Ne}$, corrected for the efficiency of the detector with a $25 \mathrm{~nm}$ foil.

energy events, in turn reducing the overall efficiency of the detector below what is shown in Fig. 4. There are two main sources of unwanted background in the spectra: multiple scattering in the substrate and random coincidences.

Multiple scattering in the substrate is a process wherein an incident ion undergoes several small-angle scattering events in the target and is steered back to the surface, just as if it had undergone a single large-angle scattering event. Because of the kinetics involved, such a particle can exit the sample with more energy than it would have had after a single large-angle scattering event. Although the probability of multiple scattering is very low, it is high enough to be a concern for the high sensitivity measurements of HIBS, since a multiple scattered particle from the bulk is indistinguishable from particles scattered directly from heavy impurities. ${ }^{16}$ Thus multiple scattering produces an unavoidable background, particularly at lower energies where the probabilities of such events are higher. To estimate the amount of this background under our conditions, one of us used TRIM ${ }^{13}$ with computational enhancements to calculate lower bounds of the multiple scattering from $\mathrm{Si}$ with various final energies. The results of these computations, which will be detailed elsewhere ${ }^{17}$, are compared in Fig. 6 to the same spectrum shown earlier in Fig. 2. The spectrum and the multiple scattering calculations are plotted as the log of differential probability versus reduced beam energy. The TRIM results are scaled by the measured detector efficiency at each energy. These calculations are not very accurate for events of such low probability, but they are in qualitative agreement with the background of the spectrum at lower 


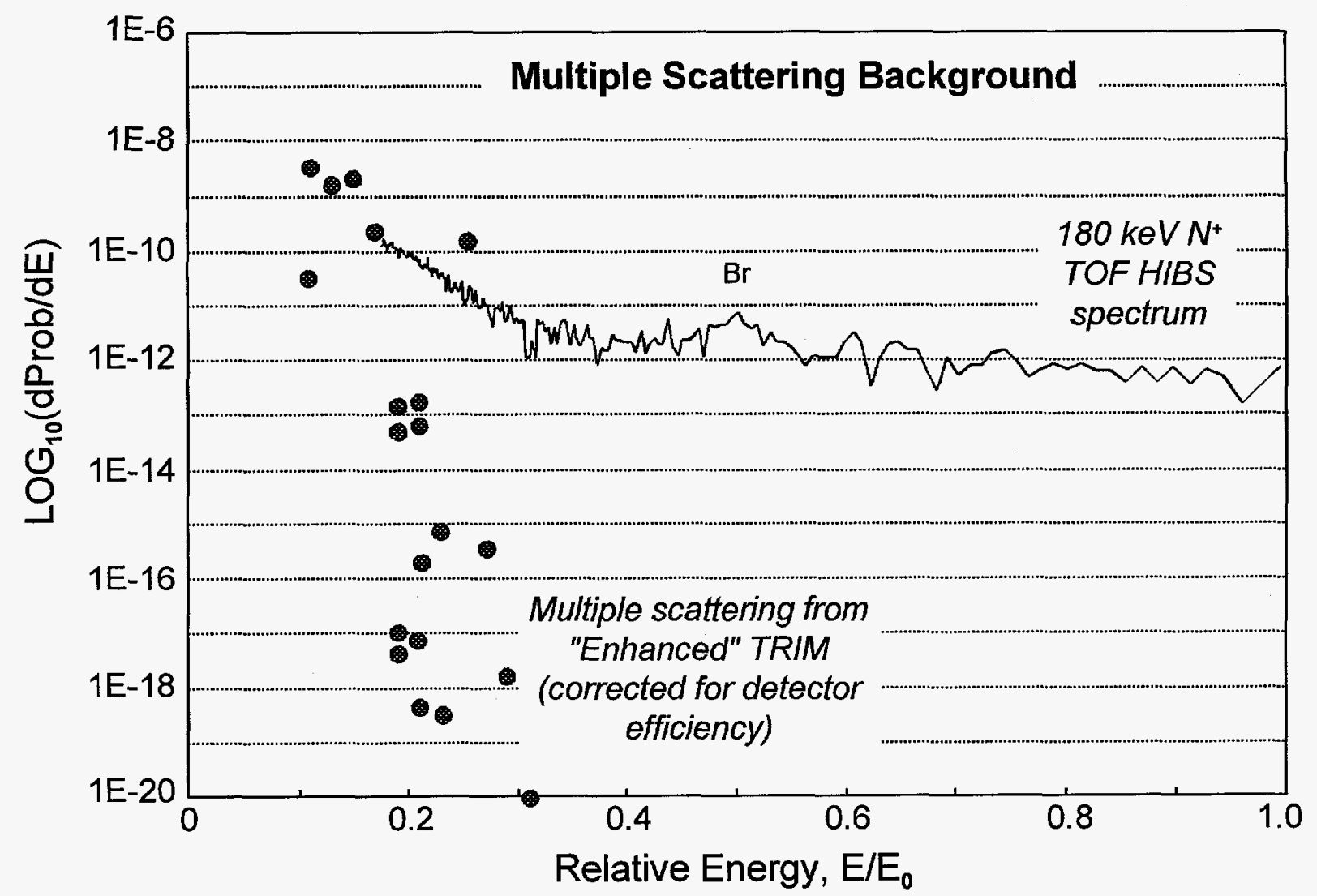

Figure 6. TRIM calculations of lower bounds for the multiple scattering from a Si substrate, for a $180 \mathrm{keV} N$ beam. The TRIM calculations, corrected for detector efficiency, are shown as filled circles. The example spectrum is shown plotted as an energy spectrum on the same scale.

energy and suggest that the direct contribution of multiple scattering to the background is insignificant for reduced energies above 0.3 .

The low level background in the remainder of the spectrum is due to random coincidences, wherein a start and stop pulse which are not physically correlated are counted as an event by the electronics. These amount to a few counts per channel for a $60 \mu \mathrm{C}$ spectrum. Although the multiple scattering is not a direct background at higher energy, the relatively large number of low energy particles from multiple scattering contribute to random coincidences. Since these particles have very low energy, they can produce electrons and a start pulse but be scattered at a large angle and escape detection by the ion MCP. This leads to a significant electron count rate without corresponding ion counts, and in turn to a higher rate of random coincidences. These appear as a low level background at all flight times. This background can be reduced by lowering the overall count rate, that is, by analyzing with a lower beam current. It can also be reduced by channeling the incident beam when the substrate is a single crystal, since that would reduce the number of multiple scattering events.

\section{SUMMARY}

A number of factors influence the sensitivity which can be achieved with HIBS; detector efficiency, choice of beam species and energy, sputtering, and backgrounds due to multiple 
scattering and random coincidences. The sensitivity can be increased in a number of ways: increasing the solid angle by using multiple detectors in parallel, using a larger beam spot, channeling the incident beam along a crystal axis of the substrate, and matching the choice of beam to the screening foil thickness. Sputtering of the sample surface by the analysis beam is the ultimate limit to the statistics which can be obtained, and hence the sensitivity. The rate of sputtering will depend on the state of the impurity: particles will sputter differently than widely dispersed, low concentration layers, so it is difficult to predict the limit in advance. However, experiments have indicated that the rate of sputtering during HIBS analysis for a sub-monolayer impurity is lower than predicted by a simple sputtering model using bulk sputtering rates. ${ }^{12}$ Using the research system with a single detector and a $25 \mathrm{~nm}$ foil, we have achieved sensitivities to impurities on Si ranging monotonically from $\sim 5 \times 10^{10}$ atoms $/ \mathrm{cm}^{2}$ for Fe to $\sim 1 \times 10^{9}$ atoms $/ \mathrm{cm}^{2}$ for $\mathrm{Au}$, without preconcentration.

\section{ACKNOWLEDGMENTS}

Technical assistance by K. G. Minor and D. L. Buller is gratefully acknowledged. The collaborations of A. C. Diebold of SEMATECH, R. A. Weller, M. H. Mendenhall, and D. Pedersen of Vanderbilt University, and G. D. Tipton and B. L. Doyle of Sandia Labs have been essential. This work was performed at Sandia National Laboratories and supported by the U.S. Department of Energy under contract no. DE-AC04-94AL85000.

\section{REFERENCES}

1. See, for example, the proceedings of Microcontamination 94, Oct. 4-6, 1994.

2. B. L. Doyle, J. A. Knapp, and D. L. Buller, Nucl. Inst. and Meth. B42, 295(1989).

3. J. A. Knapp and B. L. Doyle, Nucl. Inst. and Meth. B45, 143(1990).

4. J. A. Knapp and J. C. Banks, Nucl. Inst. and Meth. B79, 457(1993).

5. J. A. Knapp, J. C. Banks, and B. L. Doyle, Nucl. Inst. and Meth. B85, 20(1994).

6. J. A. Knapp, J. C. Banks, and B. L. Doyle, Sandia National Laboratories internal report, SAND94-0391.

7. J. A. Knapp, J. C. Banks, and A. C. Diebold, Proceedings of Microcontamination 94, ibid.

8. E. Rutherford, Phil. Mag. 21, 669(1911).

9. C. G. Darwin, Phil. Mag. 28, 499(1914).

10. S. R. Lee and R. R. Hart, Nucl. Inst. and Meth. B79, 463(1993).

11. H. H. Andersen, F. Besenbacher, P. Loftager and W. Moller, Phys. Rev. A21, 1891(1980).

12. R. A. Weller, D. Pedersen, J. H. Arps, J. C. Banks and J. A. Knapp, to be published.

13. TRIM computer code, J. F. Ziegler, IBM Research Center, Yorktown Heights, New York; See also, J. F. Ziegler, J. P. Biersack and U. Littmark, The Stopping and Range of Ions in

Solids, vol. 1 (Pegamon, New York, 1985).

14. R. A. Weller, J. H. Arps, D. Pedersen and M. H. Mendenhall, Nucl. Inst. and Meth., in press.

15. R. A. Weller, to be published.

16. D. K. Brice, Nucl. Inst. and Meth. B69, 349(1992).

17. D. K. Brice, to be published. 